\title{
Lactoferrin mRNA expression in mouse mammary glands during pregnancy and lactation
}

\author{
H.P. Li*, X.L. Jiao*, X.J. Wang, F.T. Liu, H.S. Zhu, Y.Y. Wang and \\ G.Y. Yang
}

Key Laboratory of Animal Biochemistry and Nutrition, Henan Agricultural University, Ministry of Agriculture, Zhengzhou, Henan, China

*These authors contributed equally to this study. Corresponding authors: Y.Y. Wang / G.Y. Yang

E-mail: wangyueying2008@126.com / haubiochem@163.com

Genet. Mol. Res. 13 (3): 4747-4755 (2014)

Received June 24, 2013

Accepted October 23, 2013

Published July 2, 2014

DOI http://dx.doi.org/10.4238/2014.July.2.4

\begin{abstract}
Lactoferrin (Lf) is an iron-binding glycoprotein that is produced by mucosal epithelial cells in mammals. Lf has non-immune natural defense functions and biological functions in addition to and distinct from its role in regulating inflammatory responses. Lf also improved some physiological and immunological parameters. Lf is a biomarker for monitoring medical treatment in inflammatory bowel diseases. Current LF research focuses on iron absorption, antimicrobial activity, and the modulation of iron metabolism during inflammation. No systematic research about Lf expression levels in mouse mammary glands during pregnancy and lactation exists. We investigated Lf mRNA expression levels in mouse mammary glands by collecting samples on days $1,6,12$, and 18 of pregnancy and lactation (six mice per group). The expression levels of Lf mRNA were measured by semi-quantitative reverse transcription polymerase chain reaction using GAPDH as an internal control. Lf mRNA was not expressed in mammary glands on
\end{abstract}


days 1,6 , and 12 of pregnancy, but it was expressed on day 18 (IOD: integrated optical density; $\mathrm{Lf}_{\mathrm{IOD}} / \mathrm{GAPDH}_{\mathrm{IOD}}=0.46$ ). Lf expression levels were higher during lactation stages than during pregnancy stages, and it stabilized at $0.71-0.73\left(\mathrm{Lf}_{\mathrm{IOD}} / \mathrm{GAPDH}_{\mathrm{IOD}}\right)$ from day 1 to 12 of lactation; however, the difference was not significant $(\mathrm{P}>0.05)$. At day 18 of lactation, Lf expression began to decline $\left(\mathrm{Lf}_{\mathrm{IOD}} / \mathrm{GAPDH}_{\mathrm{IOD}}=0.61\right)$, but the difference was not significant $(\mathrm{P}>0.05)$. Based on these results, the variation in Lf expression levels during developmental stages may be related to its regulatory role in mouse mammary gland immunity.

Key words: Lactoferrin; Mouse; Mammary gland; Pregnancy; Lactation

\section{INTRODUCTION}

Lactoferrin (Lf) is an iron-binding glycoprotein that is produced by mucosal epithelial cells in mammals (González-Chavez et al., 2009) and can be found in most of the exocrine secretions such as milk, tears, nasal secretions, saliva, urine, uterine secretions, and amniotic fluids (Park et al., 2011) as well as in secondary granules of neutrophils (Masson et al., 1965).

Lf has diverse functions related to its iron-binding properties, including iron absorption, antimicrobial activity, and the modulation of iron metabolism during inflammation, but it has also been found to act as an immunomodulator, transcription factor, procoagulant, ribonuclease, protease inhibitor, and protease, and it has shown anti-inflammatory and antitumoral activity (Brock, 2002). Lf has non-immune natural defense functions. The effects of Lf against microbial infections including those by parasites, bacteria, and viruses have been reported in a large number of in vivo and in vitro studies (Seganti et al., 2004). The antimicrobial activity of Lf includes activity against both Gram-positive and -negative bacteria, RNA and DNA viruses, some Candida species and other fungi, and some parasites (Tomita et al., 2002; García-Montoya et al., 2012).

However, Lf possesses biological functions in addition to and distinct from its role in regulating inflammatory responses. Mass spectrometry and Western blotting of Lf was used to diagnose periodontal diseases (Kido et al., 2012). Native bovine Lf can perform biological activities in postnatal small intestinal development (Liao et al., 2012). Paesano et al. (2012) provided strong evidence for a role of Lf in preterm delivery treatment, thus extending the therapeutic potential of this multifunctional natural protein. Besides explaining the broad anti-influenza activity of Lf, Ammendolia et al. (2012) lay the foundations for exploiting Lf fragments as a source of potential anti-influenza therapeutics. Ordaz-Pichardo et al. (2012) suggested that Lf may aid in the therapy of amoebiasis, likely without producing undesirable effects in patients. Lf was also shown to improve some physiological and immunological parameters of Siberian sturgeon to some extent (Eslamloo et al., 2012). Lf was also used as a biomarker for monitoring medical treatment in inflammatory bowel diseases (Langhorst and Boone, 2012).

Mammary gland immunity, which is defined as the protection and resistance to infectious disease, is facilitated through a variety of immune and non-immune factors. While both immune and non-immune factors interact extensively to provide adequate protection against 
mastitis, the magnitude, duration, and effectiveness of mammary gland immunity are influenced greatly by particular etiological agents (Sordillo and Streicher, 2002). To date, research on Lf has been limited simply to iron absorption, antimicrobial activity, and the modulation of iron metabolism during inflammation. There has been no systematic research on Lf expression levels in mouse mammary glands during pregnancy and lactation. In order to determine the expression of Lf mRNA in the whole developmental cycle of the mammary gland and to elucidate the impact and mechanism of Lf in mammary gland immunity during pregnancy and lactation, mouse mammary glands were used to investigate the expression of Lf mRNA during lactation by semi-quantitative reverse transcription polymerase chain reaction (RT-PCR).

\section{MATERIAL AND METHODS}

\section{Animals and sampling}

Eight-week-old Kunming mice were obtained from the Center for Laboratory Animals of Henan Province. Details of the experimental design were reported previously (Wang et al., 2011; Li et al., 2012). Briefly, a total of 8 stages of adult mouse developmental mammary glands were selected for this study: 1, 6, 12, and 18 days of pregnancy and lactation (P1, P6, P12, P18, L1, L6, L12, and L18). A total of 6 animals were used per time point.

\section{RNA extraction and reverse transcription}

Total RNA was extracted from mouse mammary glands with the TRIzol reagent kit (Invitrogen Inc., USA) and was used to determine the Lf mRNA levels by RT-PCR. All extracted RNA samples were finally dissolved in RNase-free water. The purity of the dissolved RNA was assessed by the A260/A280 nm ratio, which was measured using an ultraviolet/ visible spectrophotometer (Nanodrop 2000/2000C, USA). The RNA integrity was determined by denaturing agarose gel electrophoresis. Total RNA was extracted following a conventional protocol and was dissolved with $20 \mu \mathrm{L}$ RNase-free water.

RNA was reverse transcribed with a reaction mix containing $6.5 \mu \mathrm{L}$ diethylpyrocarbonate- $\mathrm{H}_{2} \mathrm{O}, 4 \mu \mathrm{L} 5 \mathrm{X}$ buffer, $4 \mu \mathrm{L} 2.5 \mathrm{mM}$ deoxyribonucleotide triphosphates, $1 \mu \mathrm{L} 50 \mathrm{pM}$ oligo(dT) $)_{18}, 2 \mu \mathrm{L} 5 \mathrm{U} / \mu \mathrm{L}$ AMV reverse transcriptase, $0.5 \mu \mathrm{L} 40 \mathrm{U} / \mu \mathrm{L}$ RNase inhibitor, and 2 $\mu \mathrm{L}$ RNA. The total reaction volume was $20 \mu \mathrm{L}$. After gently mixing, the solution was incubated for $60 \mathrm{~min}$ at $42^{\circ} \mathrm{C}, 15 \mathrm{~min}$ at $72^{\circ} \mathrm{C}$, and $2 \mathrm{~min}$ in an ice bath. Two controls were performed in the reverse transcription reaction: one control was prepared using all reagents except the RNA sample, for which an equivalent volume of water was substituted, and the other control was prepared using all reagents except reverse transcriptase. The controls underwent identical PCR procedures as the experimental samples. The cDNA product was stored at $-20^{\circ} \mathrm{C}$.

\section{RT-PCR}

The primers used for amplification of Lf mRNA were determined using the Primer Premier TM Version 5.0 software (PREMIER Biosoft International, Canada) and checked by basic local alignment search tool searches. All of the primers, including those for the glyceraldehyde-3-phosphate dehydrogenase (GAPDH) gene that was used as an internal 
reference, were synthesized by Shanghai Sango Biological Engineering Technology \& Services Co. Ltd. (China). Electrophoresis on $1.2 \%(\mathrm{w} / \mathrm{v})$ agarose gels was conducted to determine the quality and integrity of the primers. The sequence of primers and product size were as follows: Lf: sense 5'-tggaaggcagaagtgaaggct-3', antisense 5'-aaacaagcatcgggattccag-3', 303-bp product; and GAPDH: sense 5'-tgcaccaccaactgcttag-3', antisense 5'-gatgcagggatgatgttc-3', 175-bp product.

The cDNAs were further amplified using PCR in a $25-\mu \mathrm{L}$ mixture consisting of $1 \mu \mathrm{L}$ RT reaction solution, $12.5 \mu \mathrm{L} 2 \mathrm{X}$ Master mix (Shanghai Sango Biotechnology Company), 1 $\mu \mathrm{L} 20 \mathrm{pM}$ forward primer, $1 \mu \mathrm{L} 20 \mathrm{pM}$ reverse primer, and $9.5 \mu \mathrm{L}$ sterilized $\mathrm{H}_{2} \mathrm{O}$. The reaction substrates were mixed by gently flicking the bottom of each tube. PCR amplification was carried out for 35 cycles $\left(95^{\circ} \mathrm{C}, 30 \mathrm{~s} ; 53.6^{\circ} \mathrm{C}, 30 \mathrm{~s} ; 72^{\circ} \mathrm{C}, 30 \mathrm{~s}\right)$ for GAPDH and 30 cycles $\left(95^{\circ} \mathrm{C}, 30 \mathrm{~s} ; 56.4^{\circ} \mathrm{C}, 30 \mathrm{~s} ; 72^{\circ} \mathrm{C}, 30 \mathrm{~s}\right)$ for Lf. Reactions were completed with a final extension at $72^{\circ} \mathrm{C}$ for $10 \mathrm{~min}$. For each $5-\mu \mathrm{L}$ sample, PCR amplification products were mixed gently with $3 \mu \mathrm{L}$ fluorochrome before protection from light for $10 \mathrm{~min}$, and then they were visualized on 1.2\% agarose gels using the MultiImage Light System (Shanghai Tianneng Biotechnology Company, China).

\section{Statistical treatment of results}

All results are reported as means $\pm \mathrm{SD}$ and were analyzed by the Statistical Package for the Social Sciences (SPSS) statistical software (version 13.0). Differences between group data were analyzed using the Student-Newman-Keuls post-hoc test of one-way analysis of variance, and differences between groups were evaluated using the paired-samples $t$-test. Significance was set at $\mathrm{P}<0.05$ and $\mathrm{P}<0.01$ in two-tailed testing.

\section{RESULTS}

Gel electrophoresis showing RT-PCR of Lf in mouse mammary glands indicated that a single PCR product of about 303 bp was obtained in the P18, L1, L6, L12, and L18 samples, and the product was not observed in the P1, P6, and P12 samples (Figures 1 and 2). The relative expression levels of Lf mRNA in mouse mammary glands during pregnancy and lactation were shown in Tables 1 and 2 and Figures 3 and 4.

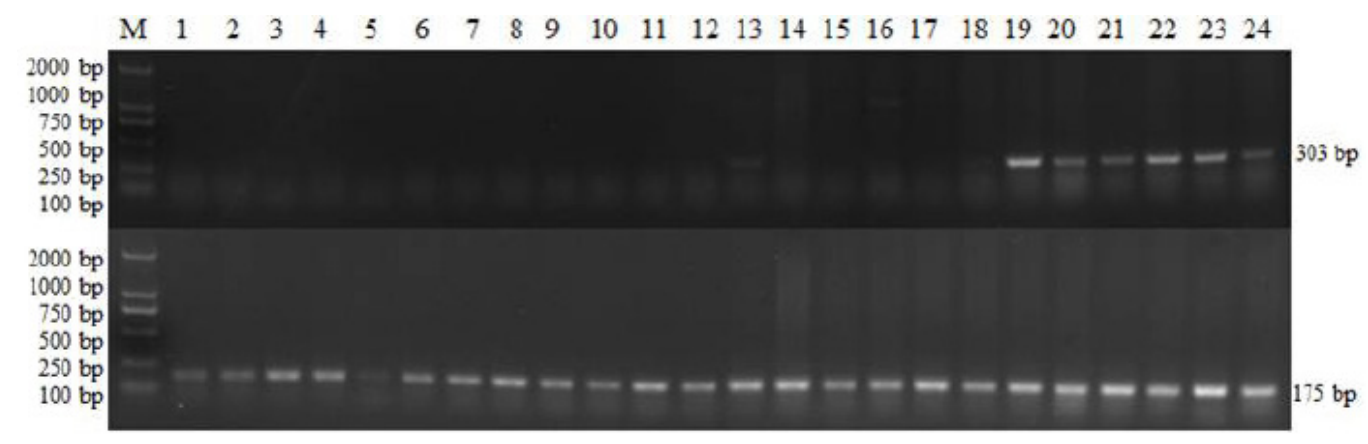

Figure 1. Gel electrophoresis of RT-PCR products in mouse mammary gland during pregnancy. Lane $M=$ marker; lanes 1-6 = P1; lanes 7-12 = P6; lanes 13-18= P12; lanes 19-24= P18. 


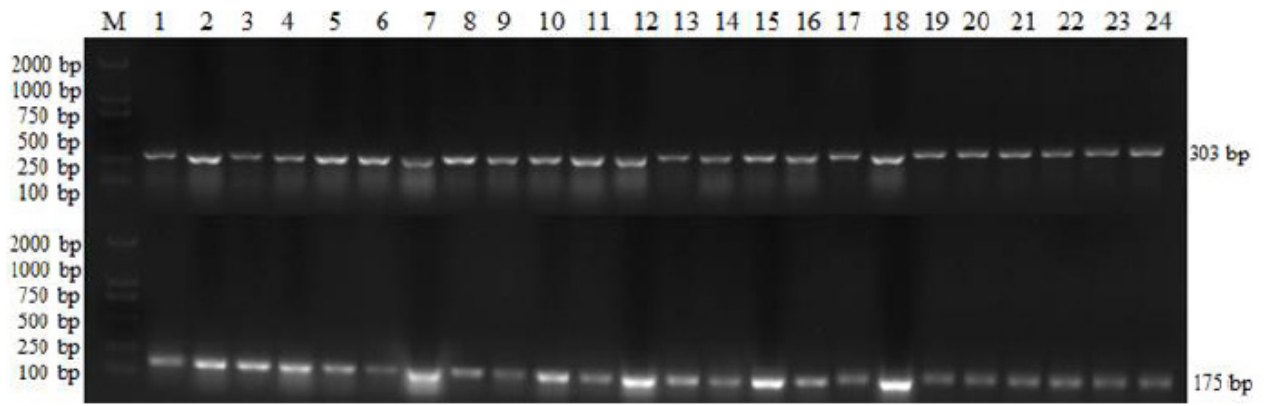

Figure 2. Gel electrophoresis of RT-PCR products in mouse mammary gland during lactation. Lane $M=$ marker; lanes 1-6= L1; lanes 7-12= L6; lanes 13-18=L12; lanes 19-24= L18.

Table 1. Expression level of lactoferrin (Lf) mRNA relative to GAPDH in mouse mammary gland during pregnancy.

\begin{tabular}{lcc}
\hline Group & No. of samples & Lf/GAPDH (IOD) \\
\hline P1 & 6 & - \\
P6 & 6 & - \\
P12 & 6 & - \\
P18 & 6 & $0.46 \pm 0.24$ \\
\hline
\end{tabular}

$(-)=$ negative. IOD $=$ integrated optical density.

Table 2. Expression level of lactoferrin (Lf) mRNA relative to GAPDH in mouse mammary gland during lactation.

\begin{tabular}{lcr}
\hline Group & No. of samples & Lf/GAPDH (IOD) \\
\hline L1 & 6 & $0.72 \pm 0.09$ \\
L6 & 6 & $0.71 \pm 0.26$ \\
L12 & 6 & $0.73 \pm 0.16$ \\
L18 & 6 & $0.61 \pm 0.16$ \\
\hline
\end{tabular}

IOD = integrated optical density.

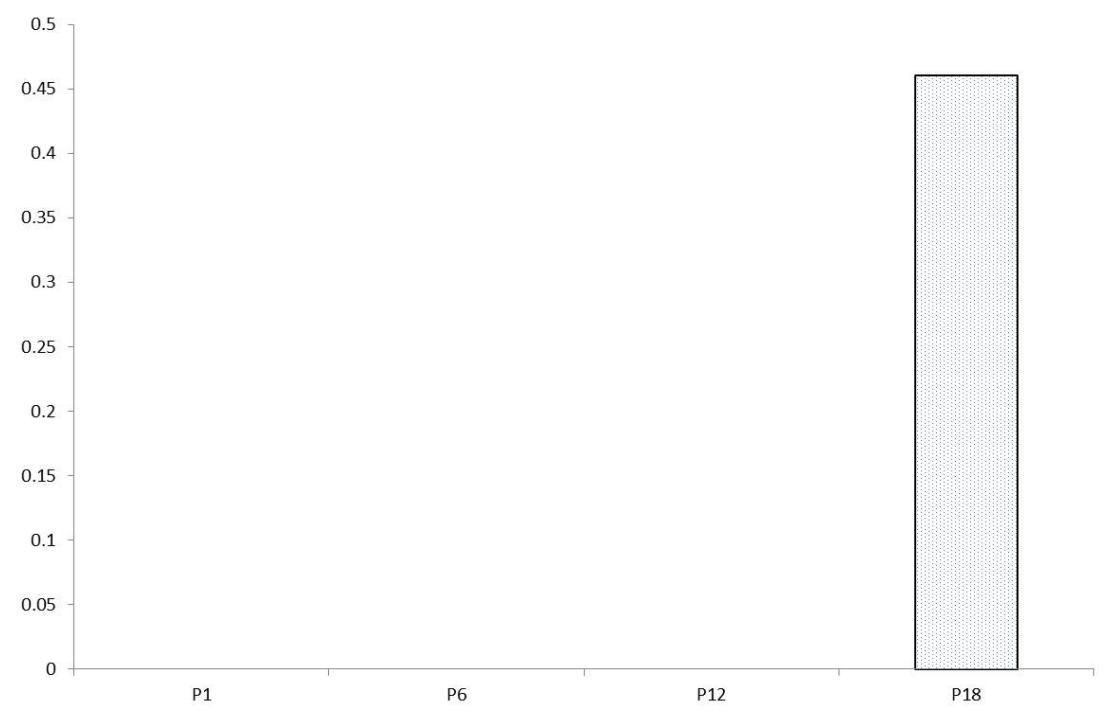

Figure 3. Expression levels of lactoferrin (Lf) mRNA relative to GAPDH in mouse mammary gland during pregnancy. 


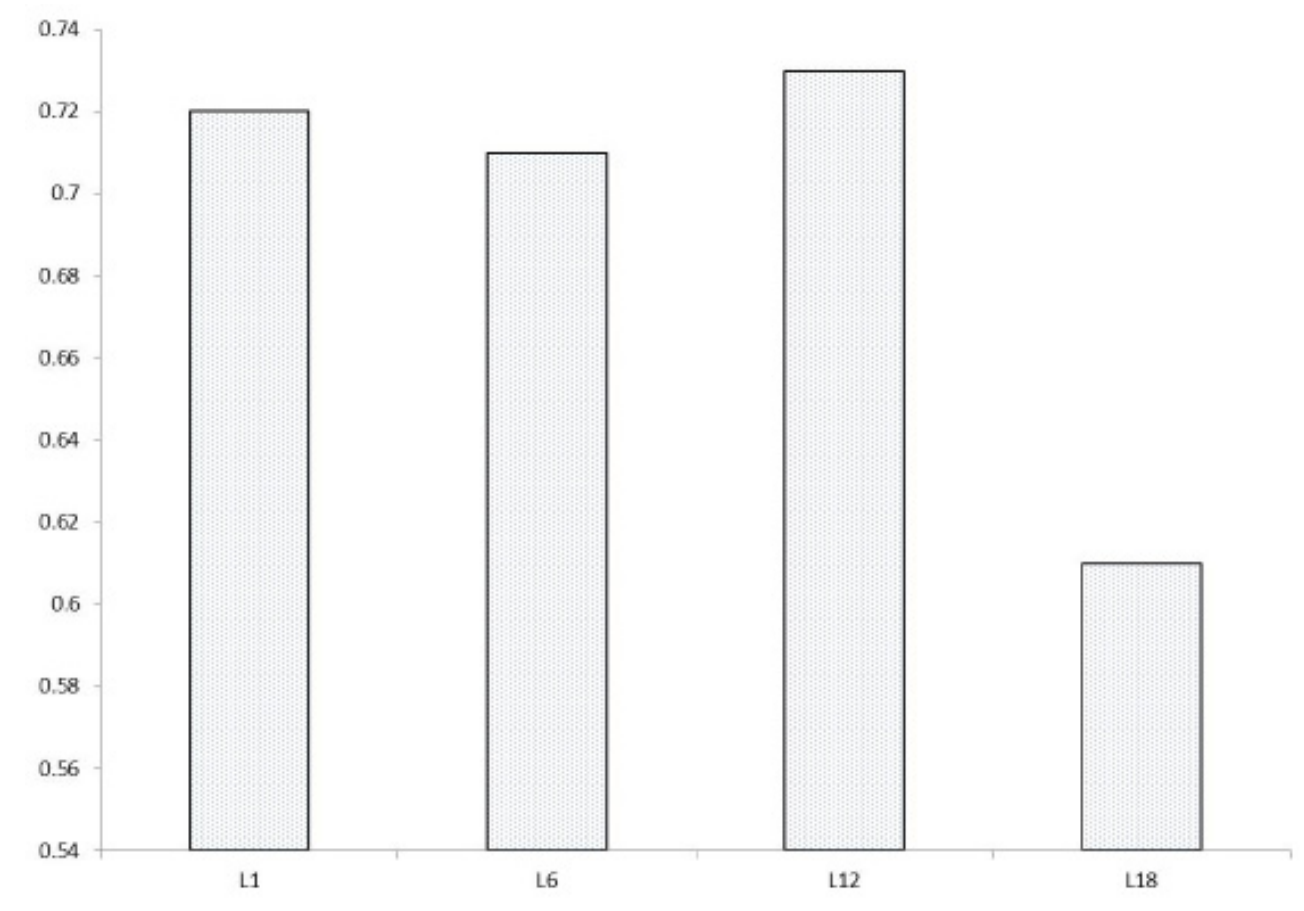

Figure 4. Expression levels of lactoferrin (Lf) mRNA relative to GAPDH in mouse mammary gland during lactation.

Lf mRNA was not expressed in the P1, P6, and P12 mammary gland samples, but it was expressed in the P18 sample (integrated optical density (IOD); $\mathrm{Lf}_{\mathrm{IOD}} / \mathrm{GAPDH}_{\mathrm{IOD}}=0.46$ ). The Lf expression levels were higher during lactation stages than during pregnancy stages. Lf expression was stabilized at $0.71-0.73\left(\mathrm{Lf}_{\mathrm{IOD}} / \mathrm{GAPDH}_{\mathrm{IOD}}\right)$ from day 1 to 12 of lactation; however, the difference was not significant $(\mathrm{P}>0.05)$. The Lf expression level declined at day 18 of lactation $\left(\mathrm{Lf}_{\mathrm{IOD}} / \mathrm{GAPDH}_{\mathrm{IOD}}=0.61\right)$, but the difference was not significant $(\mathrm{P}>0.05)$.

\section{DISCUSSION}

Both specific and innate soluble factors represent important lines of defense within the mammary gland that can elicit effective protective responses against invading pathogens (Sordillo and Streicher, 2002). The primary soluble effectors of the specific immune response are antibodies produced by antigen-activated B lymphocytes. In addition to the specific effects of antibodies, the mammary gland has nonspecific bacteriostatic factors that work together with or independent of antibodies. One such factor is Lf, an iron-binding protein produced by epithelial cells and leukocytes that functions to bind free ferric ions in milk, thereby preventing the growth of bacteria that need iron for growth (Schanbacher et al., 1993).

Accumulating evidence also indicates that Lf has several pleiotropic effects, such as the regulation of cell migration, differentiation, proliferation, and cytokine secretion. Lf is present in a variety of tissues and cell types, and its expression is under different regulatory controls. On the basis of both in vivo and in vitro studies, the transcriptional activity of the Lf 
gene is most sensitive to estrogen stimulation in the reproductive organs (Teng et al., 2002). Lf was induced by estrogen in a time- and dose-dependent fashion in the uterus of immature mice, but it was not affected by estrogen in the mammary gland. Differences were also found in the expression of Lf in the mammary glands and uterus of adult females during lactation (Teng et al., 1989).

Lf protein expression may be hindered by progesterone or some other local factor in the endometrial epithelium after ovulation. This suggests the possibility that, in addition to its known role as a secretory protein, Lf may be transported to the nucleus, serving an autocrine role (Walmer et al., 1992). The expression of $\mathrm{Lf}$ in the canine uterus during the normal estrous cycle and in bitches with pyometra was examined. Lf gene transcripts had the highest levels in estrus (Kida et al., 2006).

We found that Lf mRNA in mammary glands was not expressed during the early and middle stages of pregnancy, but we detected Lf mRNA expression in mammary glands at the end of pregnancy. During lactation stages, Lf expression levels were higher than those during pregnancy stages. Lf expression levels were stabilized from day 1 to 12 of lactation; however, this difference was not significant. Then, the Lf expression level declined in the L18 sample, but this difference was not significant. Wang et al. (2005) found that Lf mRNA was strongly expressed on day 1 of lactation, decreased gradually on days 9 and 17 of lactation, and then increased again markedly on day 25 of lactation.

Lf possesses biological functions in addition to and distinct from its role in regulating inflammatory responses. It was recently suggested that $\mathrm{Lf}$ is closely related to therapeutics (Ammendolia et al., 2012; Kido et al., 2012; Ordaz-Pichardo et al., 2012; Paesano et al., 2012), and it improved some physiological and immunological parameters (Eslamloo et al., 2012; Liao et al., 2012; Langhorst and Boone, 2012). In malignant mammary epithelial cells, Lf has been suggested to have antiproliferative effects to protect from local and distant metastasis, and downregulated Lf expression has been reported in breast cancer (Campbell et al., 1992; Benaïssa et al., 2005). Lf expression is further suggested to be associated with breast cancer susceptibility (Furmanski et al., 1989), and it is a positive prognostic indicator of breast cancer with the lobular histotype (Benaïssa et al., 2005).

Interestingly, fecal Lf increased during bacterial infection and with greater disease severity, and it may be a good marker to predict and monitor intestinal inflammation in children with infectious diarrhea (Chen et al., 2011). In particular, this biomarker was shown to be a sensitive and specific marker of disease activity in chronic inflammatory bowel diseases (Buderus et al., 2004; Judd et al., 2011). All of these studies indicate the possible use of Lf as a clinical marker of inflammatory diseases. During infection by T-cell leukemia virus type 1 and bacteria, mammary epithelial cells act as a source of secreted Lf (Zheng et al., 2005; Moriuchi and Moriuchi, 2006). The level of Lf in milk, external secretions, and serum significantly increases during infections, suggesting that this protein plays an important role in host defense against infectious agents.

We found that Lf mRNA was not expressed in mammary glands in the P1, P6, and P12 samples, but it was expressed in the P18 sample. During lactation stages, Lf expression levels were higher than those during pregnancy stages, but its expression was stabilized from days 1 to 12 of lactation; the differences in the expression were not significant. Then, the Lf expression declined in the L18 sample, but this difference was not significant. These sequential changes suggest that Lf may be transported to the nucleus, serving an autocrine role dur- 
ing developmental stages. We suggested that the variation in the Lf expression level during developmental stages may be used as a biomarker to monitor mammary gland immunity to some extent.

\section{ACKNOWLEDGMENTS}

Research supported by the Project of National Major Basic Dairy Research "973" Plan (\#2011CB100802) and by the Ministry of Agriculture (\#2011-G35).

\section{REFERENCES}

Ammendolia MG, Agamennone M, Pietrantoni A, Lannutti F, et al. (2012). Bovine lactoferrin-derived peptides as novel broad-spectrum inhibitors of influenza virus. Pathog. Glob. Health 106: 12-19.

Benaïssa M, Peyrat JP, Hornez L, Mariller C, et al. (2005). Expression and prognostic value of lactoferrin mRNA isoforms in human breast cancer. Int. J. Cancer 114: 299-306.

Brock JH (2002). The physiology of lactoferrin. Biochem. Cell Biol. 80: 1-6.

Buderus S, Boone J, Lyerly D and Lentze MJ (2004). Fecal lactoferrin: a new parameter to monitor infliximab therapy. Dig. Dis. Sci. 49: 1036-1039.

Campbell T, Skilton RA, Coombes RC, Shousha S, et al. (1992). Isolation of a lactoferrin cDNA clone and its expression in human breast cancer. Br. J. Cancer 65: 19-26.

Chen CC, Chang CJ, Lin TY, Lai MW, et al. (2011). Usefulness of fecal lactoferrin in predicting and monitoring the clinical severity of infectious diarrhea. World J. Gastroenterol. 17: 4218-4224.

Eslamloo K, Falahatkar B and Yokoyama S (2012). Effects of dietary bovine lactoferrin on growth, physiological performance, iron metabolism and non-specific immune responses of Siberian sturgeon Acipenser baeri. Fish Shellfish Immunol. 32: 976-985.

Furmanski P, Li ZP, Fortuna MB, Swamy CV, et al. (1989). Multiple molecular forms of human lactoferrin. Identification of a class of lactoferrins that possess ribonuclease activity and lack iron-binding capacity. J. Exp. Med. 170: 415-429.

García-Montoya IA, Cendon TS, Arevalo-Gallegos S and Rascon-Cruz Q (2012). Lactoferrin a multiple bioactive protein: an overview. Biochim. Biophys Acta 1820: 226-236.

González-Chávez SA, Arévalo-Gallegos S and Rascón-Cruz Q (2009). Lactoferrin: structure, function and applications. Int. J. Antimicrob. Agents 33: 301-308.

Judd TA, Day AS, Lemberg DA, Turner D, et al. (2011). Update of fecal markers of inflammation in inflammatory bowel disease. J. Gastroenterol. Hepatol. 26: 1493-1499.

Kida K, Baba E, Torii R, Kawate N, et al. (2006). Lactoferrin expression in the canine uterus during the estrous cycle and with pyometra. Theriogenology 66: 1325-1333.

Kido J, Bando M, Hiroshima Y, Iwasaka H, et al. (2012). Analysis of proteins in human gingival crevicular fluid by mass spectrometry. J. Periodontal Res. 47: 488-499.

Langhorst J and Boone J (2012). Fecal lactoferrin as a noninvasive biomarker in inflammatory bowel diseases. Drugs Today 48: 149-161.

Li HP, Guo YJ, Zhu HS, Zhong K, et al. (2012). IL-8 mRNA expression in the mouse mammary glands during pregnancy and lactation. Genet. Mol. Res. 11: 4746-4753.

Liao Y, Jiang R and Lönnerdal B (2012). Biochemical and molecular impacts of lactoferrin on small intestinal growth and development during early life. Biochem. Cell Biol. 90: 476-484.

Masson P, Heremans JF and Prignot J (1965). Immunohistochemical localization of the iron-binding protein lactoferrin in human bronchial glands. Experientia 21: 604-605.

Moriuchi M and Moriuchi H (2006). Induction of lactoferrin gene expression in myeloid or mammary gland cells by human T-cell leukemia virus type 1 (HTLV-1) tax: implications for milk-borne transmission of HTLV-1. J. Virol. 80: 7118-7126.

Ordaz-Pichardo C, León-Sicairos N, Hernández-Ramírez VI, Talamás-Rohana P, et al. (2012). Effect of bovine lactoferrin in a therapeutic hamster model of hepatic amoebiasis. Biochem. Cell Biol. 90: 425-434.

Paesano R, Pietropaoli M, Berlutti F and Valenti P (2012). Bovine lactoferrin in preventing preterm delivery associated with sterile inflammation. Biochem. Cell Biol. 90: 468-475.

Park JH, Park GT, Cho IH, Sim SM, et al. (2011). An antimicrobial protein, lactoferrin exists in the sweat: proteomic 
analysis of sweat. Exp. Dermatol. 20: 369-371.

Schanbacher FL, Goodman RE and Talhouk RS (1993). Bovine mammary lactoferrin: implications from messenger ribonucleic acid (mRNA) sequence and regulation contrary to other milk proteins. J. Dairy Sci. 76: 3812-3831.

Seganti L, Di Biase AM, Marchetti M, Pietrantoni A, et al. (2004). Antiviral activity of lactoferrin towards naked viruses. Biometals 17: 295-299.

Sordillo LM and Streicher KL (2002). Mammary gland immunity and mastitis susceptibility. J. Mammary Gland Biol. Neoplasia 7: 135-146.

Teng CT, Beard C and Gladwell W (2002). Differential expression and estrogen response of lactoferrin gene in the female reproductive tract of mouse, rat, and hamster. Biol. Reprod. 67: 1439-1449.

Teng CT, Pentecost BT, Chen YH, Newbold RR, et al. (1989). Lactotransferrin gene expression in the mouse uterus and mammary gland. Endocrinology 124: 992-999.

Tomita M, Wakabayashi H, Yamauchi K, Teraguchi S, et al. (2002). Bovine lactoferrin and lactoferricin derived from milk: production and applications. Biochem. Cell Biol. 80: 109-112.

Walmer DK, Wrona MA, Hughes CL and Nelson KG (1992). Lactoferrin expression in the mouse reproductive tract during the natural estrous cycle: correlation with circulating estradiol and progesterone. Endocrinology 131: 1458-1466.

Wang Y, Tu Y, Han F, Xu Z, et al. (2005). Developmental gene expression of lactoferrin and effect of dietary iron on gene regulation of lactoferrin in mouse mammary gland. J. Dairy Sci. 88: 2065-2071.

Wang YY, Wang YL, Li HP, Zhu HS, et al. (2011). Leptin mRNA expression in the rat mammary gland at different activation stages. Genet. Mol. Res. 10: 3657-3663.

Zheng J, Ather JL, Sonstegard TS and Kerr DE (2005). Characterization of the infection-responsive bovine lactoferrin promoter. Gene 353: 107-117. 